\title{
Field Equations and Radial Solutions in a Noncommutative Spherically Symmetric Geometry
}

\author{
Aref Yazdani \\ Department of Physics, Faculty of Basic Sciences, University of Mazandaran, P.O. Box 47416-95447, Babolsar, Iran \\ Correspondence should be addressed to Aref Yazdani; a.yazdani@stu.umz.ac.ir
}

Received 10 June 2014; Revised 6 October 2014; Accepted 19 October 2014; Published 11 November 2014

Academic Editor: Luis A. Anchordoqui

Copyright (c) 2014 Aref Yazdani. This is an open access article distributed under the Creative Commons Attribution License, which permits unrestricted use, distribution, and reproduction in any medium, provided the original work is properly cited. The publication of this article was funded by SCOAP S $^{3}$

\begin{abstract}
We study a noncommutative theory of gravity in the framework of torsional spacetime. This theory is based on a Lagrangian obtained by applying the technique of dimensional reduction of noncommutative gauge theory and that the yielded diffeomorphism invariant field theory can be made equivalent to a teleparallel formulation of gravity. Field equations are derived in the framework of teleparallel gravity through Weitzenbock geometry. We solve these field equations by considering a mass that is distributed spherically symmetrically in a stationary static spacetime in order to obtain a noncommutative line element. This new line element interestingly reaffirms the coherent state theory for a noncommutative Schwarzschild black hole. For the first time, we derive the Newtonian gravitational force equation in the commutative relativity framework, and this result could provide the possibility to investigate examples in various topics in quantum and ordinary theories of gravity.
\end{abstract}

\section{Introduction}

Field equations of gravity and radial solutions have been previously derived in noncommutative geometry [1-5]. The generalization of quantum field theory by noncommutativity based on coordinate coherent state formalism also cures the short distance behavior of point-like structures [613]. In this method, the particle mass $M$, instead of being completely localized at a point, is dispensed throughout a region of linear size $\sqrt{\theta}$, substituting the position Dirac-delta function, describing point-like structures, with a Gaussian function, and describing smeared structures. In other words, we assume that the energy density of a static, spherically symmetric, particle-like gravitational source cannot be a delta function distribution and will be given by a Gaussian distribution of minimal width $\sqrt{\theta}$ as follows:

$$
\rho_{\theta}(r)=\frac{M}{(4 \pi \theta)^{3 / 2}} \exp \left(-\frac{r^{2}}{4 \theta}\right)
$$

Furthermore, noncommutative gauge theory appears in string theory [14-18]: the boundary theory of an open string is noncommutative when it ends on D-bran with a constant
B-field or an Abelian gauge field (particularly see [14]). Therefore, closed string theories are expected to remain commutative as long as the background is geometric. Recent evidence has found a connection between nongeometry and closed string noncommutativity and even nonassociativity [19-21]; approaches using dual membrane theories [22] and matrix models $[23,24]$ arrive at the same conclusion.

The ordinary quantum field theory is unable to present an exact description of exotic effects of the inherent nonlocality of interactions, so we need a model to provide an effective description of many of the nonlocal effects in string theory within a simpler setting [25].

The model leads to the gauge theories of gravitation through an ordinary class of dimensional reductions of noncommutative electrodynamics on flat space, which then can be made equivalent to a formulation of teleparallel gravity, macroscopically describing general relativity. Moreover, this model is developed by the parallel theories of gravitation, giving a clear understanding of Einstein's principle of absolute parallelism. It is defined by a nontrivial vierbein field and formed by a linear connection. For carrying nonvanishing torsion, this connection is known as Wietzenböck geometry on spacetime. 
This model is given appropriately by a noncommutative Lagrangian and introduced by authors in $[4,5]$. Admittedly, this Lagrangian and the relevant explanations will be the basis of our next general calculations. In this paper are going to use the Greek alphabet $(\mu, \nu, \rho, \ldots=0,1,2,3)$ to denote indices related to spacetime and the first half of the Latin alphabet $(a, b, c, \ldots=0,1,2,3)$ to denote indices related to the tangent space. A Minkowski spacetime whose Lorentz metric is assumed to have the form of $\eta_{a b}=\operatorname{diag}(-1,+1,+1,+1)$. The middle letters of the Latin alphabet $(i, j, k, \ldots=1,2,3)$ will be reserved for space indices. The noncommutative Lagrangian is expressed as

$$
\begin{aligned}
\dot{L}_{G r}= & \frac{\chi_{0}}{e^{2}} \operatorname{det}\left(h_{\sigma}^{\sigma^{\prime}}\right) \eta^{\mu \mu^{\prime}} \\
& \times\left[\frac{1}{4} \eta^{\nu \nu^{\prime}} \eta_{\lambda \lambda^{\prime}} \dot{T}_{\mu \nu}^{\lambda} \dot{T}_{\mu^{\prime} \nu^{\prime}}^{\lambda^{\prime}}-\dot{T}_{\mu \nu}^{\nu} \dot{T}_{\mu^{\prime} \nu^{\prime}}^{v^{\prime}}+\frac{1}{2} \dot{T}_{\mu \nu}^{\nu^{\prime}} \dot{T}_{\mu^{\prime} \nu^{\prime}}^{\nu}\right] .
\end{aligned}
$$

In the usual way, having a Lagrangian, which describes gravitation based on noncommutative background, is like those of gauge theories written in terms of contractions of its field strength, here represented by torsion of Weitzenböck connection. Its behavior under a local change of $\Delta_{\mu}$ is the main invariance property of the particular combination torsion tensor fields. Here $e$ is Yang-Mills coupling constant, noncommutative scale determines the Planck length, and the Planck scale of $n$-dimensional spacetime is given by

$$
k=\sqrt{16 \pi G_{N}}=e\left|\operatorname{Pfaff}\left(\Theta^{A B}\right)\right|^{1 / 2 n} .
$$

In mass dimension 2 the weight constant $\chi_{0}$ is

$$
\chi_{0}=\left|P f a f f\left(\Theta^{A B}\right)\right|^{-1 / n} .
$$

In the commutative limit, it reduces to gravitational constant. Therefore, $\Theta^{A B}$ is a noncommutative parameter, defined as

$$
\Theta^{A B}=\left(\begin{array}{ll}
\theta^{\mu \nu} & \theta^{\mu b} \\
\theta^{\mu b} & \theta^{a b}
\end{array}\right) \longrightarrow \theta^{\mu \nu}=\theta^{a b}=0 .
$$

By considering the calculation of superpotential and energymomentum current with respect to noncommutative gauge potential, given by $B_{a}^{\mu}=\left|\operatorname{det}\left(\theta^{\mu^{\prime} a^{\prime}}\right)\right|^{1 / 2 n} \widehat{\theta}^{v \mu} \omega_{a v}$, the version of non-commutative gravitational field equations is produced. $\omega_{a v}$ are gauge fields corresponding to the gauging of the translation group, that is, replacing $R^{n}$ by the Lie algebra $g$ of local gauge transformations with gauge functions and its relation with the non-trivial tetrad field is expressed as $h_{a}^{\mu}=\delta_{a}^{\mu}-e \theta^{\nu \mu} \omega_{a v}$ and $\delta_{a}^{\mu}$ has the perturbative effect in the trivial holonomic tetrad fields of flat space.

It is important to note that by applying the "dimensional reduction of gauge theories," noncommutative electrodynamics gauge field, shown by the noncommutative YangMiles theory, reduces to the gauge theories of gravitation, which naturally yields Weitzenböck geometry on the spacetime. Also, the induced diffeomorphism invariant field theory can be made equivalent to a teleparallel formulation of gravity macroscopically describing general relativity. In Section 2 we show that our Lagrangian can be made equivalent with general relativity. In Section 3 we are going to derive the field equations by utilizing various definitions of teleparallel gravity. By simplifying and solving the field equations, we obtain the line element in the spherically symmetric spacetime in Section 4. We continue our discussion with investigations about the limiting cases of our line element and horizons of noncommutative Schwarzschild black hole in this method. Finally we show how the Newtonian gravitational force equation can be derived from our line element in the commutative limit in Section 5.

\section{Equivalence with General Relativity}

In order to continue our discussion to achieve to noncommutative field equations, we should show how our model can be coupled with general relativity. With respect to the given relation of

$$
\dot{\Gamma}_{\mu \nu}^{\rho}=\Gamma_{\mu \nu}^{\rho}+\dot{K}_{\mu \nu}^{\rho}
$$

for the vanishing curvature of the Weitzenböck connection, we have

$$
\dot{R}_{\theta \mu \nu}^{\rho}=R_{\theta \mu \nu}^{\rho}+\dot{Q}_{\theta \mu \nu}^{\rho} \equiv 0
$$

where

$$
R_{\theta \mu \nu}^{\rho}=\partial_{\mu} \Gamma_{\theta \nu}^{\rho}-\partial_{\nu} \Gamma_{\theta \nu}^{\rho}+\Gamma_{\sigma \mu}^{\rho} \Gamma_{\theta \nu}^{\sigma}-\Gamma_{\sigma \nu}^{\rho} \Gamma_{\theta \mu}^{\sigma}
$$

is the curvature of the Levi-Civita connection. The above equations show that, whereas in general relativity torsion vanishes, in teleparallel gravity it is curvature that vanishes. We rewrite (7) based on their components in order to find the scaler of $\dot{R}_{\theta \mu \nu}^{\rho}$; therefore, we have

$$
\begin{aligned}
\dot{Q}_{\theta \mu \nu}^{\rho}=( & \partial_{\mu} \dot{K}_{\theta \nu}^{\rho}-\partial_{\nu} \dot{K}_{\theta \mu}^{\rho}+\dot{T}_{\sigma \mu}^{\rho} \dot{K}_{\theta \nu}^{\sigma}-\dot{\Gamma}_{\sigma \nu}^{\rho} \dot{K}_{\theta \mu}^{\sigma} \\
& \left.\quad \dot{\Gamma}_{\theta \mu}^{\sigma} \dot{K}_{\sigma \nu}^{\rho}+\dot{\Gamma}_{\theta \nu}^{\sigma} \dot{K}_{\sigma \mu}^{\rho}\right)+\dot{K}_{\sigma \nu}^{\rho} \dot{K}_{\theta \mu}^{\sigma}-\dot{K}_{\sigma \mu}^{\rho} \dot{K}_{\theta \nu}^{\sigma},
\end{aligned}
$$

that is, the tensor written in terms of the Weitzenböck connection only. Like the Riemanian curvature tensor, it is 2form assuming values in the Lie algebra of the Lorentz group (see $[26,27])$. By taking appropriate contractions it is easy to show that

$$
\dot{Q}_{\theta \mu \nu}^{\rho}=\left(\dot{D}_{\mu} \dot{k}_{\theta \nu}^{\rho}-\dot{D}_{\nu} \dot{k}_{\theta \mu}^{\rho}\right)+\dot{K}_{\sigma \nu}^{\rho} \dot{K}_{\theta \mu}^{\sigma}-\dot{K}_{\sigma \mu}^{\rho} \dot{K}_{\theta \nu}^{\sigma} \text {. }
$$

By considering (20) and the following term:

$$
-R=\dot{Q} \equiv \frac{1}{2} \Lambda_{\rho}^{\theta} \dot{Q}_{\theta \mu \nu}^{\rho} d x^{\mu} \wedge d x^{\nu}
$$

we achieve to the scalar version of (7),

$$
R \equiv\left(\dot{K}^{\mu \nu \rho} \dot{K}_{\rho \nu \mu}-\dot{K}_{\mu \rho}^{v} \dot{K}_{\nu}^{\mu \rho}\right)+\frac{2}{h} \partial_{\mu}\left(h \dot{T}_{\nu}^{\nu \mu}\right) .
$$

The Lagrangian of (2) can be written in a simple form of

$$
\dot{L}=\frac{\chi_{0}}{e^{2}} \operatorname{det}\left(h_{\sigma}^{\sigma^{\prime}}\right)\left(\dot{K}^{\mu \nu \rho} \dot{K}_{\rho \nu \mu}-\dot{K}_{\mu \rho}^{v} \dot{K}_{\nu}^{\mu \rho}\right)
$$


with a combination of (12) and (13), where $\dot{L}$ takes the following form:

$$
\dot{L}=\frac{\chi_{0}}{e^{2}} \operatorname{det}\left(h_{\sigma}^{\sigma^{\prime}}\right)\left(R-\frac{2}{h}\left(\partial_{\mu}\left(h \dot{T}_{\nu}^{\nu \mu}\right)\right)\right) .
$$

By considering (3) and (4), $\dot{L}$ exchanges to

$$
\dot{L}=L-\partial_{\mu}\left(\frac{h}{8 \pi G} \dot{T}_{\nu}^{\nu \mu}\right),
$$

up to a divergence at the commutative limit; therefore, the Lagrangian of (2) $\dot{L}$ is equivalent to the Lagrangian of general relativity

$$
\dot{L}=\frac{-1}{16 \pi G} \sqrt{-g} R
$$

is the Einstein-Hilbert Lagrangian of general relativity. However, this result could be extended with many further terms, but this is enough to derive a valid field equations.

\section{Noncommutative Field Equations}

In this section, we are going to present a reformulation of teleparallel gravity (which is made equivalent to general relativity). Due to the introduced noncommutative Lagrangian (2), we are able to derive the field equations similarly to the teleparallel method. Weitzenböck geometric definitions and some well-known concepts of general relativity [28-30] and teleparallel gravity are required (more explanations about these equations can be found in $[29,31,32])$. In 4-dimension, the noncommutative action integral is given by

$$
S=\int \dot{L}_{G r} d^{4} x
$$

Under an arbitrary variation $\delta h_{a}^{\mu}$ of the tetrad field, the action variation is written in the following form:

$$
\delta S=\int \Xi_{\mu}^{a} \delta h_{a}^{\mu} h d^{4} x,
$$

where

$$
h \Xi_{\mu}^{a}=\frac{\delta \dot{L}_{G r}}{\delta B_{a}^{\mu}} \equiv \frac{\delta \dot{L}_{G r}}{\delta h_{a}^{\mu}}=\frac{\partial \dot{L}_{G r}}{\partial h_{a}^{\mu}}-\partial_{\lambda} \frac{\partial \dot{L}_{G r}}{\partial_{\lambda} \partial h_{a}^{\mu}}
$$

is the matter energy-momentum tensor. (More definitions about this tensor can be found in [33].) Now, consider first an infinitesimal Lorentz transformation as

$$
\Lambda_{a}^{b}=\delta_{a}^{b}+\varepsilon_{a}^{b}
$$

where $\varepsilon_{a}^{b}=-\varepsilon_{a}^{b}$. Because of such transformation, the tetrad should be changed as

$$
\delta h_{a}^{\mu}=\varepsilon_{a}^{b} h_{b}^{\mu} .
$$

The requirement of invariance of the action under local Lorentz transformation therefore yields

$$
\int \Xi_{a}^{b} \varepsilon_{a}^{b} h d^{4} x=0
$$

Since $\varepsilon_{a}^{b}$ is antisymmetric, symmetric of energy-momentum tensor yields some specific results that can be seen in [28]. Consider spacetime coordinates that are transformed as follows:

$$
x^{\prime \rho}=x^{\rho}+\zeta^{\rho} .
$$

Whereby, we retrieve the tetrad in the form of

$$
\delta h_{a}^{\mu} \equiv h_{a}^{\prime \mu}(x)-h_{a}^{\mu}(x)=h_{a}^{\rho} \partial_{\rho} \zeta^{\mu}-\zeta^{\rho} \partial_{\rho} h_{a}^{\mu} .
$$

Substituting in (18), we have

$$
\delta S=\int \Xi_{\mu}^{a}\left[h_{\rho}^{a} \partial_{\mu} \zeta^{\rho}-\zeta^{\rho} \partial_{\rho} h_{a}^{\mu}\right] h d^{4} x,
$$

or equivalently

$$
\delta S=\int\left[\Xi_{c}^{\rho} \partial_{\rho} \zeta^{c}+\zeta^{c} \Xi_{\mu}^{\rho} \partial_{\rho} h_{c}^{\mu}-\zeta^{\rho} \partial_{\rho} h_{a}^{\mu}\right] h d^{4} x .
$$

Substitute the identity

$$
\partial_{\rho} h_{a}^{\mu}=\dot{A}_{a \rho}^{b} h_{b}^{\mu}-\dot{\Gamma}_{\lambda \rho}^{\mu} h_{a}^{\lambda},
$$

where $\dot{A}$ is the spin connection in teleparallel gravity. The important property of teleparallel gravity is that its spin connection is related only to the inertial properties of the frame, not to gravitation. In fact, it is possible to choose an appropriate frame in which it vanishes everywhere. We know that the above formula vanishes by (41) (see also $[34,35]$ ), and making use of the symmetric of the energy-momentum tensor, the action variation assumes the form of

$$
\delta S=\int \Xi_{c}^{\rho}\left[\partial_{\rho} \zeta^{c}+\left(\dot{A}_{b \rho}^{c}-\dot{K}_{b \rho}^{c}\right) \zeta^{b}\right] h d^{4} x
$$

Integrating the first term by parts and neglecting the surface term, the invariance of the action yields

$$
\int\left[\partial_{\mu}\left(h \Xi_{a}^{\mu}\right)-\left(\dot{A}_{a \mu}^{b}-\dot{K}_{a \mu}^{b}\right)\left(h \Xi_{b}^{\mu}\right)\right] \zeta^{a} h d^{4} x=0 .
$$

From arbitrariness of $\zeta^{c}$, under the covariant derivative $\ddot{D}_{\mu}$, it follows that

$$
\ddot{D}_{\mu} h \Xi_{a}^{\mu} \equiv \partial_{\mu}\left(h \Xi_{a}^{\mu}\right)-\left(\dot{A}_{a \mu}^{b}-\dot{K}_{a \mu}^{b}\right)\left(h \Xi_{b}^{\mu}\right)=0 .
$$

By identity of

$$
\partial_{\rho} h=h \dot{\Gamma}_{\nu \rho}^{v} \equiv h\left(\dot{\Gamma}_{\rho \nu}^{v}-\dot{K}_{\rho \nu}^{v}\right),
$$

the above conservation law becomes

$$
\partial_{\mu} \Xi_{a}^{\mu}+\left(\dot{\Gamma}_{\rho \mu}^{\mu}-\dot{K}_{\rho \mu}^{\mu}\right) \Xi_{a}^{\rho}-\left(\dot{A}_{a \mu}^{b}-\dot{K}_{a \mu}^{b}\right) \Xi_{b}^{\mu}=0 .
$$

In a purely spacetime form, it reads

$$
\ddot{D}_{\mu} \Xi_{\lambda}^{\mu} \equiv \partial_{\mu} \Xi_{\lambda}^{\mu}+\left(\dot{\Gamma}_{\mu \rho}^{\mu}-\dot{K}_{\rho \mu}^{\mu}\right) \Xi_{\lambda}^{\rho}-\left(\dot{\Gamma}_{\lambda \mu}^{\rho}-\dot{K}_{\lambda \mu}^{\rho}\right) \Xi_{\rho}^{\mu}=0 .
$$


This is the conservation law of the source of energymomentum tensor. Variation with respect to the noncommutative gauge potential $B_{a}^{\mu}$ yields the noncommutative teleparallel version of the gravitational field equations

$$
\partial_{\sigma}\left(h \dot{S}_{a}^{\mu \sigma}\right)-k h \dot{J}_{a}^{\mu}=k h \Xi_{a}^{\mu},
$$

where

$$
h \dot{S}_{a}^{\mu \sigma}=h h_{a}^{\lambda} \dot{S}_{\lambda}^{\mu \sigma} \equiv-k \frac{\partial \dot{L}}{\partial\left(\partial_{\sigma} h_{\mu}^{a}\right)},
$$

which defines the superpotential. For the gauge current we have

$$
h \dot{J}_{a}^{\mu}=-\frac{\partial \dot{L}}{\partial B_{\mu}^{a}} \equiv-\frac{\partial \dot{L}}{\partial h_{\mu}^{a}} .
$$

Note that the matter energy-momentum tensor which is defined in this relation appears as the source of torsion; similarly, the energy-momentum tensor appears as the source of curvature in general relativity. Our computation has led us to the following results:

$$
\begin{aligned}
& \dot{S}_{a}^{\mu \sigma}=2 \dot{T}_{a}^{\mu \sigma}-\dot{T}_{a}^{\sigma \mu}-h_{a}^{\sigma} \dot{T}_{\eta}^{\eta \mu}+h_{a}^{\mu} \dot{T}_{\eta}^{\eta \sigma}, \\
& \dot{J}_{a}^{\mu}=\frac{1}{k} h_{a}^{\lambda} \dot{S}_{c}^{\nu \mu} \dot{T}_{\nu \lambda}^{c}-\frac{h_{a}^{\mu}}{h} \dot{L}+\frac{1}{k} \dot{A}_{a \sigma}^{c} \dot{S}_{c}^{\mu \sigma},
\end{aligned}
$$

for noncommutative superpotential and gauge current. The lagrangian $\dot{L}$ appears again in our equations, but notice that this term cross its coefficient yields a term purely based on its field strength according to (2). This simplified expression maintains equivalence to general relativity. We can observe that the gravitational field equations depend on the torsion only. Finally the field equations can be written as

$$
\begin{aligned}
\partial_{\sigma} & \left(h\left(2 \dot{T}_{a}^{\mu \sigma}-\dot{T}_{a}^{\sigma \mu}-h_{a}^{\sigma} \dot{T}_{\eta}^{\eta \mu}+h_{a}^{\mu} \dot{T}_{\eta}^{\eta \sigma}\right)\right) \\
- & k h\left(\frac{1}{k} h_{a}^{\lambda} \dot{S}_{c}^{\nu \mu} \dot{T}_{\nu \lambda}^{c}-\frac{h_{a}^{\mu}}{h} \dot{L}+\frac{1}{k} \dot{A}_{a \sigma}^{c} \dot{S}_{c}^{\mu \sigma}\right)=k h \Xi_{a}^{\mu},
\end{aligned}
$$

where $k=\chi_{0} / e^{2}$ is a constant. These field equations are similar to teleparallel field equations, although it would be distinguished with different field strength $\dot{T}_{a}^{\mu \sigma}$ which is given by the covariant rotational of noncommutative gauge potential of $B_{a}^{\mu}$. Consider the following equations from the teleparallel theory (see, for instance, [32, 34-36]):

$$
\begin{gathered}
\dot{T}_{\mu \nu}^{a}=\partial_{\nu} h_{\mu}^{a}-\partial_{\mu} h_{\nu}^{a}+\dot{A}_{e v}^{a} h_{\mu}^{e}-\dot{A}_{e \mu}^{a} h_{\nu}^{e}, \\
\dot{\Gamma}_{\nu \mu}^{\rho}=h_{a}^{\rho} \partial_{\mu} h_{\nu}^{a}+h_{a}^{\rho} \dot{A}_{b \mu}^{a} h_{\nu}^{b}, \\
\partial_{\mu} h_{\nu}^{a}-\dot{\Gamma}_{\nu \mu}^{\rho} h_{\rho}^{a}+\dot{A}_{b \mu}^{a} h_{\nu}^{b}=0, \\
\dot{T}_{\nu \mu}^{\rho}=\dot{\Gamma}_{\mu \nu}^{\rho}-\dot{\Gamma}_{\nu \mu}^{\rho} .
\end{gathered}
$$

The field equations take the exact following form:

$$
\begin{aligned}
& \frac{\partial}{\partial x^{\sigma}}\left(\dot{\Gamma}_{a \mu}^{\sigma}-\dot{\Gamma}_{\mu a}^{\sigma}\right)-\frac{\partial}{\partial x^{\mu}} \dot{\Gamma}_{a \lambda}^{\lambda}+\frac{\partial}{\partial x^{\lambda}} \dot{\Gamma}_{a \mu}^{\lambda} \\
& -\dot{\Gamma}_{a \lambda}^{\eta} \dot{\Gamma}_{\mu \eta}^{\lambda}+\dot{\Gamma}_{a \mu}^{\eta} \dot{\Gamma}_{\lambda \eta}^{\lambda}=\frac{\chi_{0}}{e^{2}} \rho(r) \frac{\partial}{\partial x^{a}} \frac{\partial}{\partial x^{\mu}},
\end{aligned}
$$

which, unlike the left hand side of (38), is written purely based on noncommutative field strength, and the above field equation is written in terms of Weitzenböck connection only. Regarding the equivalency between corresponding Lagrangians and the above simplified field equations and applying (34), we have therefore

$$
R_{a \mu}-\frac{1}{2} h_{a \mu} R=k \Xi_{a \mu}
$$

as equivalent to Einstein's field equations. Note that (44) is not Einstein's field equations but the teleparallel field equations made equivalent to general relativity. And equivalent model of teleparallel field equations with general relativity is expressed in the references in detail (see, for instance, [32, 34, 35]). We continue our discussion to derive noncommutative line element by solving these field equations.

\section{Noncommutative Line Element}

Teleparallel versions of the stationary, static, spherically, axissymmetric, and symmetric of the Schwarzschild solution have been previously obtained $[37,38]$. Within a framework inspired by noncommutative geometry, we solve the field equations for a distribution of spherically symmetrically mass in a stationary static spacetime, like the exterior solution of Schwarzschild (see also [29]). Then it is natural to assume that the line element is as follows:

$$
d s^{2}=-f(\widetilde{r}) d t^{2}+g(\widetilde{r}) d r^{2}+h(\widetilde{r}) \widetilde{r}^{2}\left(d \theta^{2}+\sin ^{2} \theta d \phi^{2}\right) .
$$

With a new radial coordinate defined as $r=\tilde{r} \sqrt{h(\widetilde{r})}$, the line element becomes

$$
d s^{2}=-A(r) d t^{2}+B(r) d r^{2}+r^{2}\left(d \theta^{2}+\sin ^{2} \theta d \phi^{2}\right) .
$$

Usually one replaces the functions $A(r)$ and $B(r)$ with exponential functions to obtain somewhat simpler expressions for the noncommutative tensor components. Hence, we introduce the functions $\alpha(r)$ and $\beta(r)$ by $e^{2 \alpha(r)}=A(r)$ and $e^{\beta(r)}=B(r)$ to get

$$
d s^{2}=-e^{2 \alpha} d t^{2}+e^{2 \beta} d r^{2}+r^{2}\left(d \theta^{2}+\sin ^{2} \theta d \phi^{2}\right)
$$

Tetrad components of the above metric take the following form:

$$
h_{\mu}^{a}=\left[\begin{array}{cccc}
-e^{2 \alpha} & 0 & 0 & 0 \\
0 & e^{2 \beta} \sin \theta \cos \phi & r \cos \theta \cos \phi & -r \cos \theta \sin \phi \\
0 & e^{2 \beta} \sin \theta \sin \phi & r \cos \theta \sin \phi & r \sin \theta \cos \phi \\
0 & e^{2 \beta} \cos \theta & -r \sin \theta & 0
\end{array}\right] .
$$

Weitzenböck connection $\dot{\Gamma}_{\mu \nu}^{\rho}$ has the following expression:

$$
\dot{\Gamma}_{\mu \nu}^{\rho}=h_{a}^{\rho} \partial_{\nu} h_{\mu}^{a}
$$


Now, we can calculate the nonvanishing components of Weitzenböck connection as follows:

$$
\begin{gathered}
\Gamma_{01}^{0}=-2 \alpha^{\prime}, \quad \Gamma_{11}^{1}=2 \beta^{\prime}, \quad \Gamma_{22}^{1}=-r e^{-\beta}, \\
\Gamma_{33}^{1}=-r e^{-\beta} \sin ^{2} \theta, \quad \Gamma_{12}^{2}=\frac{e^{\alpha}}{r}=\Gamma_{13}^{3}, \\
\Gamma_{21}^{2}=\frac{1}{r}=\Gamma_{31}^{3}, \quad \Gamma_{33}^{2}=-\sin \theta \cos \theta, \\
\Gamma_{23}^{3}=\Gamma_{32}^{3}=\cot \theta .
\end{gathered}
$$

By replacing these components in (43), the noncommutative tensors of (51)-(53) for the left-hand side of the field equations will produce the following expression:

$$
\begin{gathered}
N_{\overparen{t} t}=\frac{1}{r^{2}}\left(-4 e^{-2 \beta}+1-\psi_{\theta}\right)-\frac{2}{r} \beta^{\prime} e^{-2 \beta}=\frac{\chi_{0}}{e^{2}} \rho(r) \delta_{\overparen{t} \hat{t}}, \\
N_{\widehat{r} \widehat{r}}=\frac{1}{r^{2}}\left(2 e^{-2 \beta}+1-\psi_{\theta}\right)+\frac{2}{r} \alpha^{\prime} e^{-2 \beta}=\frac{\chi_{0}}{e^{2}} \rho(r) \delta_{\widehat{r} \hat{r}}, \\
N_{\widehat{\theta} \widehat{\theta}}=N_{\widehat{\phi} \hat{\phi}}=\frac{1}{r} e^{-2 \beta}\left(r \alpha^{\prime \prime}+r \alpha^{\prime 2}-r \alpha^{\prime} \beta^{\prime}+\alpha^{\prime}-\beta^{\prime}-1\right) \\
+\alpha^{\prime 2} e^{-2 \beta}=\frac{\chi_{0}}{e^{2}} \rho(r) \delta_{\widehat{\theta} \widehat{\theta}}=\frac{\chi_{0}}{e^{2}} \rho(r) \delta_{\widehat{\phi} \hat{\phi}} .
\end{gathered}
$$

Adding (51) and (52) we get simply

$$
\frac{1}{r^{2}}\left(\psi_{\theta}-e^{-2 \beta}+e^{-2 \beta}\left(\alpha^{\prime}-\beta^{\prime}\right)+1\right)=\frac{\chi_{0}}{e^{2}} \rho(r),
$$

where $\alpha(r) \neq \beta(r)$. It should also be noted that, by recalling (47), we can consider the limiting case for our solution assuming $\left(\alpha^{\prime}-\beta^{\prime}\right)=k$, where $k$ is a constant, and by considering the time coordinate, we can shift this constant to an arbitrary value. It is possible, therefore, without loss of generality to choose $k=0$. It does not contradict (47) to set $\alpha^{\prime}=\beta^{\prime}$. According to this analysis, the equation $N_{\widetilde{t} t}=$ $\left(\chi_{0} / e^{2}\right) \rho(r) \delta_{\overparen{t} t}$ can be written as

$$
\frac{-1}{r} \frac{d}{d r}\left[r\left(e^{-2 \beta}-\psi_{\theta}-1\right)\right]=\frac{\chi_{0}}{e^{2}} \rho(r) .
$$

For a perfect fluid in thermodynamic equilibrium, the stressenergy tensor takes on a particularly simple form

$$
\Xi^{\mu \nu}=(\rho+P) u^{\mu} u^{\nu}+p g^{\mu \nu},
$$

where the pressure $P$ can be neglected due to the distribution of mass and the gravitational effects; consequently, only one term will remain in the above formula as follows:

$$
\Xi^{a \mu}=\rho(r) \frac{d x^{a}}{d t} \frac{d x^{\mu}}{d t},
$$

or

$$
\Xi^{a \mu}=\rho(r) \delta^{a \mu} .
$$

Therefore, for spherically symmetric distribution of mass that depends on r-coordinate, we can write

$$
m(r)=\int_{0}^{r} 4 \pi r^{2} \rho(r) d r .
$$

Note that the $\rho(r)$ is defined by (1). Indeed, we introduce the same energy density indicated in the noncommutative perturbation theory [39]

$$
m(r)=M_{\theta}(r)=\frac{2 M}{\sqrt{\pi}} \gamma\left(\frac{3}{2}, \frac{r^{2}}{4 \theta}\right) .
$$

Equation (55) can be integrated to find

$$
e^{-2 \beta}=1-\frac{\chi_{0}}{4 \pi e^{2}} \frac{m(r)}{r}+\psi_{\theta},
$$

where $\psi_{\theta}$ is a function that carries the tetrad field factor and will be defined later by (66) and (67). Now by considering

$$
e^{-2 \beta}=-\frac{1}{h_{11}}=h_{00},
$$

the noncommutative line element for a spherically symmetric matter distribution is therefore

$$
\begin{aligned}
d s^{2}= & -\left(1-\frac{\chi_{0}}{4 \pi e^{2}} \frac{m(r)}{r}+\psi_{\theta}\right) d t^{2} \\
& +\left(1-\frac{\chi_{0}}{4 \pi e^{2}} \frac{m(r)}{r}+\psi_{\theta}\right)^{-1} d r^{2} \\
& +r^{2}\left(d \theta^{2}+\sin ^{2} \theta d \phi^{2}\right) .
\end{aligned}
$$

The constant field of $\chi_{0} / e^{2}$ in terms of (3), (4), and (5) can be retrieved as

$$
\frac{\chi_{0}}{e^{2}}=\frac{\left|\theta^{\mu b}\right|}{16 \pi G_{N}},
$$

where $G_{N}$ is the Newtonian constant and $\left|\theta^{\mu b}\right|$ is determined by $\theta^{\mu b}=\theta^{21}=-\theta^{12} \equiv \theta$. Where, $\theta$ is a real, antisymmetric and constant tensor, therefore, the above equation can be simplified to yield:

$$
\frac{\chi_{0}}{e^{2}}=\frac{\theta}{16 \pi G_{N}} .
$$

New line element (63) in particular depends on $\psi_{\theta}$, and naturally $\psi_{\theta}$ has its origin on the quantum fluctuations of the noncommutative background geometry and originally comes from the field equations. The presented solution for our field equations produces naturally some additional terms in comparison with the solution of noncommutative version of general relativity (naturally, because it has some additional terms in its components). These terms appear in the new line element because $\psi_{\theta}$ relates to the noncommutative torsional spacetime and algebraic properties in spherically symmetric solution of the tetrad fields. We have therefore $\psi_{\theta}$ in the following simplified equation:

$$
\psi_{\theta}=\varepsilon^{\widehat{r} \hat{\theta} \widehat{\phi}} \varepsilon_{\widehat{r} \hat{\theta} \hat{\phi}} h_{\widehat{r}}^{\widehat{r}} e^{-\beta}
$$

Definition $\varepsilon^{\widehat{r} \widehat{\theta} \widehat{\phi}} \varepsilon_{\widehat{r} \hat{\theta} \hat{\phi}}=-6 / h^{2}$ is applied here (see also $[40,41])$. According to this definition and (48) and (50). 
Through simplification, we find the following form of $\psi_{\theta}$ we find the following form of $\psi_{\theta}$ :

$$
\psi_{\theta} \cong \sum_{k=2 n} \sum_{n=1}\left(\frac{\chi_{0}}{4 \pi e^{2}} \frac{m(r)}{r}\right)^{k}-\sum_{k=2 n+1} \sum_{n=1}\left(\frac{\chi_{0}}{4 \pi e^{2}} \frac{m(r)}{r}\right)^{k} .
$$

Note that $\psi_{\theta}$ is considered with the lower bound of $\sum$. If we want to consider at least the second order of $\theta$ (which is proposed by [13]) for $\psi_{\theta}$, then it is natural to assume $n=1$. Therefore, two states for our line element will be produced: the imperfect state and the perfect state. Let us now consider the perfect state. There is a proof for this state in terms of some theorems in mathematics that allows us to introduce our line element as an appropriate description for a noncommutative spacetime. Combination of these theorems with regard to our results is given by [42-47].

Theorem 1. Let L be a perfect field. Recall that a polynomial $f(x) \in L[x]$ is called additive if $f(x+y)=f(x)+f(y)$ identically. It is easy to see that a polynomial is additive if and only if it is of the form

$$
\begin{aligned}
f(x) & =1-a_{0} x+a_{1}^{2} x^{2}-\cdots \pm a_{n}^{n} x^{n} \\
& =\sum_{n=0} a_{n}^{2 n} x^{2 n}-\sum_{n=0} a_{n}^{2 n+1} x^{2 n+1} .
\end{aligned}
$$

The set of additive polynomials forms a noncommutative field in which $(f \circ g)(x)=f(g(x))$. This field is generated by scalar multiplications $x \mapsto$ ax for $a \in L$ and $x_{i} \in f(x)$ does not commute with the $x_{j} \in f(x)$. Note that a can be a constant field and it has given as $\approx \chi_{0} / 4 \pi e^{2}$ here (see [42-47] and the references cited therein). It is clear that components of $f(x)$ can be exactly replaced with components of $h_{00}$.

Regarding other investigations into descriptions of noncommutative spacetime, we should expand our discussion into a comparison method with other line elements presented for noncommutative spacetime. Reference [13] suggests the following line element for noncommutative Schwarzschild spacetime:

$$
\begin{aligned}
d s^{2}= & -\left(1-\frac{4 M}{r \sqrt{\pi}} \gamma\left(\frac{3}{2}, \frac{r^{2}}{4 \theta}\right)\right) d t^{2} \\
& +\left(1-\frac{4 M}{r \sqrt{\pi}} \gamma\left(\frac{3}{2}, \frac{r^{2}}{4 \theta}\right)\right)^{-1} d r^{2}+r^{2} d \Omega^{2},
\end{aligned}
$$

where $d \Omega^{2}=d \theta^{2}+\sin ^{2} \theta d \phi^{2}$ and $\gamma\left(3 / 2, r^{2} / 4 \theta\right)$ is the lower incomplete gamma function

$$
\gamma\left(\frac{3}{2}, \frac{r^{2}}{4 \theta}\right) \equiv \int_{0}^{r^{2} / 4 \theta} d t \sqrt{t} e^{-t}
$$

We note that nonvanishing radial pressure is a consequence of the quantum fluctuation of the spacetime manifold leading to an inward gravitational pull and preventing the matter collapsing into a point. According to the line element (63), in a neighborhood of the origin at $r \leq \theta$, the energy density distribution of a static symmetric and noncommutative fuzzy spacetime is described by (1), which replaces the Dirac $\delta$ distribution by a smeared Gaussian profile. Meanwhile, in the imperfect state, our line element can be made equivalent to the line element of (69), and it is expected to happen when $\psi_{\theta}$ vanishes. Assuredly it is due to vanishing of the tetrad components $h_{\mu}^{a}$ in (48) or even Wietzenböck connections in (50). It means that, in absence of torsional spacetime, the coordinate coherent state will be produced in the noncommutative field theory. It is completely reasonable since coherent state theory is derived in the noncommutative framework of the general relativity, and the torsion is not defined in the general relativity. This equivalency is shown with the following relation:

$$
1-\frac{M}{2 r \sqrt{\pi}} \gamma\left(\frac{3}{2}, \frac{r^{2}}{4 \theta}\right) \cong g_{00}^{\text {coherent state }}=1-\frac{4 M}{r \sqrt{\pi}} \gamma\left(\frac{3}{2}, \frac{r^{2}}{4 \theta}\right) .
$$

According to this proof, the solution of the presented noncommutative field equations in the imperfect state of itself results in the exact solution of noncommutative general relativity field equations through coordinate coherent state of our line element.

4.1. Schwarzschild Black Hole, Horizons. In this paper we have not extended our discussion into black holes, but our introduced equations can be the basis of a subject on noncommutative black holes. Indeed the calculation of event horizons of a noncommutative Schwarzschild black hole would be done by the horizon equation $-h_{r_{H}}=h^{11}\left(r_{H}\right)=0$. Answers to this equation are illustrated in Figures 1 and 2. Figure 1 shows the behavior of $h_{00}$ versus the horizon radii when $\psi_{\theta}$ vanishes. It is clear that $\psi_{\theta}$ vanishing approximately results in $g_{00}$ of (69); Figure 2 shows the behavior of $h_{00}$ at the same conditions when we have $\psi_{\theta}$. As we can see from these figures, there is a different behavior in the perfect state in comparison with the imperfect state near the horizon radii which is due to the nature of torsional spacetime. However, the same behaviors have been indicated in the origin and the higher bound of $r$.

\section{Force Equation in Commutative Limit}

In teleparallel gravity, the Newtonian force equation is obtained by assuming the class of frames in which the teleparallel spin connection $\dot{A}$ vanishes, and the gravitational field is stationary and weak [40, 41, 48, 49]. In our model, the Newtonian gravitational force equation directly derives from torsion components by $\psi_{\theta}$ in its commutative limit. When we write the expansion of new line element in the noncommutative limit, we have

$$
h_{00}=1-\frac{\chi_{0}}{4 e^{2}} \frac{m(r)}{r}+\left(\frac{\chi_{0}}{4 e^{2}} \frac{m(r)}{r}\right)^{2}-\left(\frac{\chi_{0}}{4 e^{2}} \frac{m(r)}{r}\right)^{3}
$$




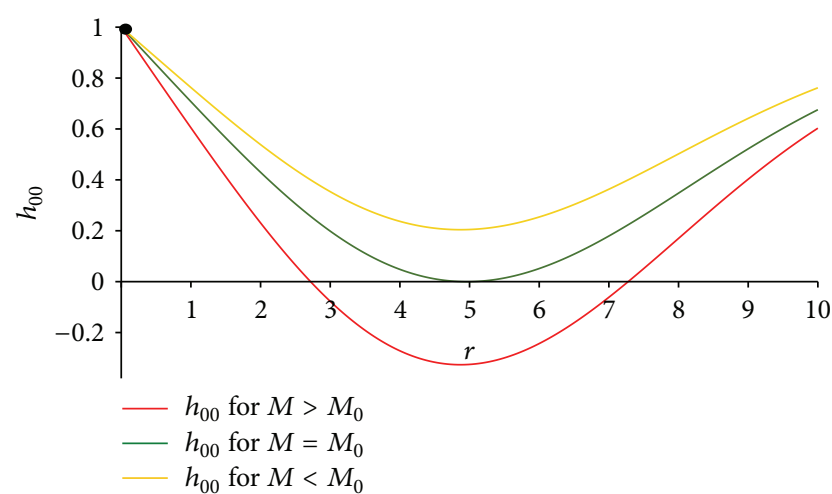

FIGURE 1: The imperfect state in a noncommutative spherically symmetric geometry. The function of $h_{00}$ versus $r \sqrt{\theta}$, for various values of $M \sqrt{\theta}$. The upper curve corresponds to $M=1.00 \sqrt{\theta}$ (without horizon), the middle curve corresponds to $M=M_{0} \approx$ $1.90 \sqrt{\theta}$ (with one horizon at $r_{H}=r_{0} \approx 4.9 \sqrt{\theta}$ ), and finally the lowest curve corresponds to $M=3.02 \sqrt{\theta}$ (two horizons at $r_{H}=$ $r_{-} \approx 2.70 \sqrt{\theta}$ and $\left.r_{H}=r_{+} \approx 7.20 \sqrt{\theta}\right)$.

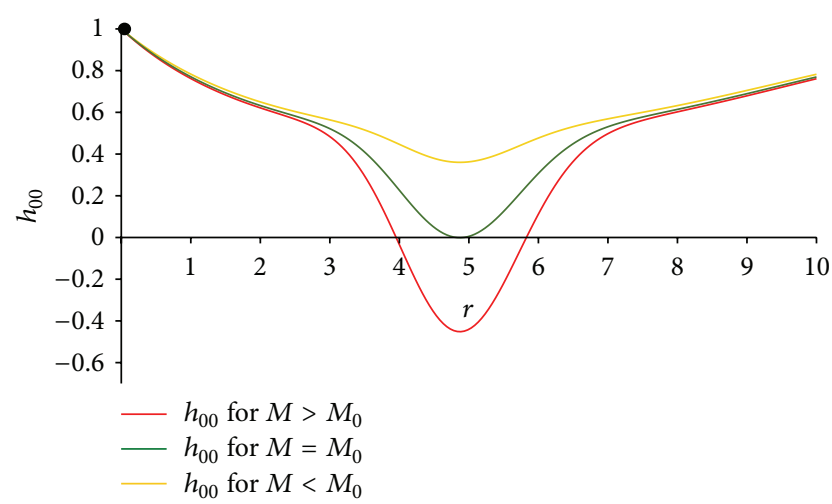

FIGURE 2: The perfect state in a noncommutative spherically symmetric geometry. The function of $h_{00}$ versus $r \sqrt{\theta}$, for various values of $M \sqrt{\theta}$. The upper curve corresponds to $M=1.00 \sqrt{\theta}$ (without horizon), the middle one corresponds to $M=M_{0} \approx 1.90 \sqrt{\theta}$ (with one horizon at $r_{H}=r_{0} \approx 4.9 \sqrt{\theta}$ ), and finally the lowest curve corresponds to $M=3.02 \sqrt{\theta}$ (two horizons at $r_{H}=r_{-} \approx 3.90 \sqrt{\theta}$ and $r_{H}=r_{+} \approx 5.80 \sqrt{\theta}$ ).

or equivalently

$$
h_{00}=1-A \frac{m(r)}{r}+B \frac{m(r)^{2}}{r^{2}}-C \frac{m(r)^{3}}{r^{3}} .
$$

Due to noncommutative effects, $r$ in the denominator vanishes, but in the limit case, when it goes to the commutative limit, it is modified to the commutative $g_{00}$ of Schwarzschild solution in addition to a force equation much similar to the Newtonian gravitational force equation. Note that the induced gravitational constant of (3) vanishes in the commutative limit and agrees with that found in [50] using the supergravity dual of noncommutative Yang-Mills theory in four dimensions. Newton was the first to consider in his Principia an extended expression of his law of gravity including an inverse-cube term of the form

$$
F=G \frac{m_{1} m_{2}}{r^{2}}+B \frac{m_{1} m_{2}}{r^{3}}, \quad B \text { is a constant. }
$$

He attempts to explain Moon's apsidal motion by the above relation. In the commutative limit our metric can be defined in the form of

$$
h_{00}^{\text {commutative }} \cong 1-\frac{2 M}{r}+\frac{4 M^{2}}{r^{2}}-\frac{8 M^{3}}{r^{3}}
$$

where $m(r)$ is given by (60), and in the commutative limit it has the form of

$$
\lim _{\theta \rightarrow 0} m(r)=2 M
$$

By considering the following terms in (74):

(i) relativistic limits $G=1$,

(ii) set the $m_{1}=m_{2}=2 M, B=-2 M$,

for our line element we can set

$$
h_{00}^{\text {commutative }}=\left(g_{00}^{\text {commutative Schwarszchild solution }}+F(r)^{\text {Newton }}\right) \text {. }
$$

As we can see from (63) and (67) (expansion of new line element), the $h_{00}$ has two parts: torsional and nontorsional parts; the above relation states that, in the limit of commutativity, torsional parts reduce to force equation of $F(r)$ and nontorsional part yields the $g_{00}$ of commutative Schwarzschild solution.

Einstein's theory of general relativity attributes gravitation to curved spacetime instead of being due to a force propagated between bodies. Energy and momentum distort spacetime in their vicinity, and other particles move in trajectories determined by the geometry of spacetime. Therefore, descriptions of the motions of light and mass are consistent with all available observations. Meanwhile, according to the general relativity's definition, the gravitational force is a fictitious force due to the curvature of spacetime because the gravitational acceleration of a body in free fall is due to its world line being a geodesic of spacetime [51]. However, through a weak equivalence principle that is assumed initially in teleparallel gravity [52-54], our results are reasonable and we can conclude that the presented solution in its commutative limit attributes the gravitation to a force propagated between bodies and the curved spacetime, or sum of torsion and curvature. This result is similar to Einstein-Cartan theory of gravity [55].

Moreover, the different behaviour of Schwarzschild black hole horizon, which is absent in the previous method, is due to force of heavy pulling from the black hole in terms of this introduced force. As can be seen in Figure 2, the intensity of this force has a direct relation with mass $M$, so the heavier the black hole is, the stronger force it has near its horizon. 


\section{Conclusion}

In this letter, we have utilized a noncommutative Lagrangian which gives us possibilities to use teleparallel gravity to derive field equations. Solution of these field equations in the spherically symmetric geometry yields a new noncommutative line element. In the limit cases when the torsion vanishes, we have obtained an interesting result: in absence of torsional spacetime the version of coordinate coherent state in noncommutative field theory will be produced. Incidentally, Figures 1 and 2 show other limit cases in our solution at the large distances and different range of masses.

As we expressed before, there are conceptual differences, in general relativity, curvature is used to geometrize the gravitational interaction, geometry replaces the concept of force, and the trajectories are determined, not by force equations but by geodesics. Teleparallel gravity, on the other hand, attributes gravitation to torsion. Torsion, however, accounts for gravitation not by geometrizing the interaction but by acting as a force $[40,41]$. This is a definition used in teleparallel gravity, whereas our model does not exactly coincide with teleparallel gravity (in the limit case only); therefore, it is natural to have more complex results especially that the definition of the existing force in torsion for gravitational interactions is approved clearly in the limit of commutativity in our model. Absolutely, attributing the gravitation to the force equation in the relativity framework which is shown directly through the commutative limit of our line element can be utilized in the various branches of physics.

\section{Conflict of Interests}

The author declares that there is no conflict of interests regarding the publication of this paper.

\section{Acknowledgment}

The author would like to thank Mr. Arya Bandari for effective proofreading of this paper.

\section{References}

[1] M. R. Douglas and N. A. Nekrasov, "Noncommutative field theory," Reviews of Modern Physics, vol. 73, no. 4, pp. 977-1029, 2001.

[2] W. Kalau and M. Walze, "Gravity, non-commutative geometry and the Wodzicki residue," Journal of Geometry and Physics, vol. 16, no. 4, pp. 327-344, 1995.

[3] P. Nicolini, "Noncommutative black holes, the final appeal to quantum gravity: a review," International Journal of Modern Physics A, vol. 24, no. 7, pp. 1229-1308, 2009.

[4] R. J. Szabo, "Quantum field theory on noncommutative spaces," Physics Reports, vol. 378, no. 4, pp. 207-299, 2003.

[5] E. Langmann and R. J. Szabo, "Teleparallel gravity and dimensional reductions of noncommutative gauge theory," Physical Review D, vol. 64, no. 10, Article ID 104019, 15 pages, 2001.

[6] X. Calmet and A. Kobakhidze, "Noncommutative general relativity," Physical Review D, vol. 72, no. 4, Article ID 045010, 2005.
[7] X. Calmet and A. Kobakhidze, "Second order noncommutative corrections to gravity," Physical Review D: Particles, Fields, Gravitation and Cosmology, vol. 74, no. 4, Article ID 047702, 2006.

[8] A. H. Chamseddine, "Deforming Einstein's gravity," Physics Letters B, vol. 504, no. 1-2, pp. 33-37, 2001.

[9] P. Aschieri, C. Blohmann, M. Dimitrijević, F. Meyer, P. Schupp, and J. Wess, "A gravity theory on noncommutative spaces," Classical and Quantum Gravity, vol. 22, no. 17, p. 3511, 2005.

[10] A. Smailagic and E. Spallucci, "Feynman path integral on the noncommutative plane," Journal of Physics A, vol. 36, p. L467, 2003.

[11] A. Smailagic and E. Spallucci, "UV divergence-free QFT on noncommutative plane," Journal of Physics A: Mathematical and General, vol. 36, no. 39, pp. L517-L521, 2003.

[12] A. Smailagic and E. Spallucci, "Lorentz invariance, unitarity and UV-finiteness of QFT on noncommutative spacetime," Journal of Physics A: Mathematical and General, vol. 37, no. 28, pp. 71697178, 2004.

[13] P. Nicolini, A. Smailagic, and E. Spallucci, "Noncommutative geometry inspired Schwarzschild black hole," Physics Letters. B, vol. 632, no. 4, pp. 547-551, 2006.

[14] C.-S. Chu and P.-M. Ho, "Non-commutative open string and Dbrane," Nuclear Physics B, vol. 550, no. 1-2, pp. 151-168, 1999.

[15] N. Seiberg and E. Witten, "String theory and noncommutative geometry," The Journal of High Energy Physics, vol. 9, article 32, 1999.

[16] F. Ardalan, H. Arfaei, and M. Sheikh-Jabbari, "Noncommutative geometry from strings and branes," Journal of High Energy Physics, vol. 2, p. 16, 1999.

[17] F. Ardalan, H. Arfaei, and M. M. Sheikh-Jabbari, "Dirac quantization of open strings and noncommutativity in branes," Nuclear Physics B, vol. 576, no. 1-3, pp. 578-596, 2000.

[18] C. Sochichiu, "M[any] vacua of IIB," Journal of High Energy Physics, vol. 2000, no. 5, article 026, 12 pages, 2000.

[19] R. Blumenhagen and E. Plauschinn, "Nonassociative gravity in string theory?" Journal of Physics A: Mathematical and Theoretical, vol. 44, no. 1, Article ID 015401, 19 pages, 2011.

[20] D. Lust, "T-duality and closed string non-commutative (doubled) geometry," Journal of High Energy Physics, vol. 2010, article $84,2010$.

[21] C. Condeescu, I. Florakis, and D. Lüst, "Asymmetric orbifolds, non-geometric fluxes and non-commutativity in closed string theory," Journal of High Energy Physics, vol. 2012, no. 4, p. 121, 2012.

[22] C. Sämann and R. J. Szabo, "Groupoids, loop spaces and quantization of 2-plectic manifolds," Reviews in Mathematical Physics, vol. 25, no. 3, Article ID 1330005, 72 pages, 2013.

[23] A. Konechny and A. Schwarz, "Introduction to M(atrix) theory and noncommutative geometry," Physics Reports, vol. 360, no. 5-6, pp. 353-465, 2002.

[24] D. Mylonas, P. Schupp, and R. J. Szabo, "Membrane sigmamodels and quantization of non-geometric flux backgrounds," Journal of High Energy Physics, vol. 2012, article 12, 2012.

[25] A. Chatzistavrakidis and L. Jonke, "Matrix theory origins of non-geometric fluxes," Journal of High Energy Physics, vol. 2013, no. 2, article 040, 2013.

[26] P. Nicolini, "Vacuum energy momentum tensor in $(2+1)$ NC scalar field theory," In press, http://arxiv.org/abs/hep-th/ 0401204. 
[27] A. Gruppuso, "Newton's law in an effective non-commutative space-time," Journal of Physics A, vol. 38, no. 9, pp. 2039-2042, 2005.

[28] S. Ansoldi, P. Nicolini, A. Smailagic, and E. Spallucci, "Noncommutative geometry inspired charged black holes," Physics Letters B, vol. 645, no. 2-3, pp. 261-266, 2007.

[29] D. McMahon, Relativity Demystified, Tata McGraw-Hill Education, 2006.

[30] Ø. Grøn and S. Hervik, Einstein's General Theory of Relativity: With Modern Applications in Cosmology, Springer, 2007.

[31] S. Weinberg, Gravitation and Cosmology, vol. 126, John Wiley \& Sons, New York, NY, USA, 1972.

[32] V. C. de Andrade, L. C. T. Guillen, and J. G. Pereira, “Teleparallel gravity: an overview," in Proceedings of the 9th Marcel Grossmann Meeting, pp. 1022-1023, July 2000.

[33] D. I. Olive, Lectures on Gauge Theories and Lie Algebras, University of Virginia, Charlottesville, Va, USA, 1982, notes taken by G. Bhattacharya and N. Turok.

[34] J. W. Maluf, M. V. Veiga, and J. F. da Rocha-Neto, "Regularized expression for the gravitational energy-momentum in teleparallel gravity and the principle of equivalence," General Relativity and Gravitation, vol. 39, no. 3, pp. 227-240, 2007.

[35] J. W. Maluf, "The teleparallel equivalent of general relativity," Annalen der Physik, vol. 525, no. 5, pp. 339-357, 2013.

[36] J. G. Pereira, "Teleparallelism: a new insight into gravity," http://arxiv.org/abs/1302.6983.

[37] M. Sharif and M. J. Amir, "Teleparallel Killing vectors of the Einstein universe," Modern Physics Letters A: Particles and Fields, Gravitation, Cosmology, Nuclear Physics, vol. 23, no. 13, pp. 963-969, 2008.

[38] J. G. Pereira, T. Vargas, and C. M. Zhang, "Axial-vector torsion and the teleparallel Kerr spacetime," Classical and Quantum Gravity, vol. 18, no. 5, pp. 833-841, 2001.

[39] K. Nozari and A. Yazdani, "The energy distribution of a noncommutative Reissner-Nordström black hole," Chinese Physics Letters, vol. 30, no. 9, Article ID 090401, 2013.

[40] R. Aldrovandi and J. G. Pereira, Teleparallel Gravity: An Introduction, vol. 173, Springer, New York, NY, USA, 2013.

[41] V. C. de Andrade and J. G. Pereira, "Gravitational Lorentz force and the description of the gravitational interaction," Physical Review D: Particles, Fields, Gravitation and Cosmology, vol. 56, no. 8, pp. 4689-4695, 1997.

[42] D. Goss, "The adjoint of the Carlitz module and Fermat's last theorem," Finite Fields and Their Applications, vol. 1, no. 2, pp. 165-188, 1995.

[43] D. S. Thakur, "Hypergeometric functions for function fields," Finite Fields and Their Applications, vol. 1, no. 2, pp. 219-231, 1995.

[44] D. Goss, Basic Structures of Function Field Arithmetic, Springer, New York, NY, USA, 1997.

[45] K. S. Kedlaya, "The algebraic closure of the power series field in positive characteristic," Proceedings of the American Mathematical Society, vol. 129, no. 12, pp. 3461-3470, 2001.

[46] H. Hironaka, "Resolution of singularities of an algebraic variety over a field of characteristic zero: II," Annals of Mathematics, vol. 79, pp. 205-326, 1964.

[47] B. Poonen, "Fractional power series and pairings on Drinfeld modules," Journal of the American Mathematical Society, vol. 9, no. 3, pp. 783-812, 1996.

[48] G. Zet, "Schwarzschild solution on a space-time with torsion," http://arxiv.org/abs/gr-qc/0308078.
[49] H. I. Arcos and J. G. Pereira, "Torsion gravity: a reappraisal," International Journal of Modern Physics D, vol. 13, no. 10, pp. 2193-2240, 2004.

[50] N. Ishibashi, S. Iso, H. Kawai, and Y. Kitazawa, "String scale in noncommutative Yang-Mills," Nuclear Physics: B, vol. 583, no. 1-2, pp. 159-181, 2000.

[51] http://en.wikipedia.org/wiki/Newton\%27s_law_of_universal_ gravitation.

[52] G. J. Olmo, "Violation of the equivalence principle in modified theories of gravity," Physical Review Letters, vol. 98, no. 6, Article ID 061101, 2007.

[53] R. Aldrovandi, J. G. Pereira, and K. H. Vu, "Gravitation without the equivalence principle," General Relativity and Gravitation, vol. 36, no. 1, pp. 101-110, 2004.

[54] C. Brans and R. H. Dicke, "Mach's principle and a relativistic theory of gravitation," Physical Review, vol. 124, no. 3, pp. 925935, 1961.

[55] A. Trautman, "Einstein-Cartan theory," http://arxiv.org/abs/grqc/0606062. 

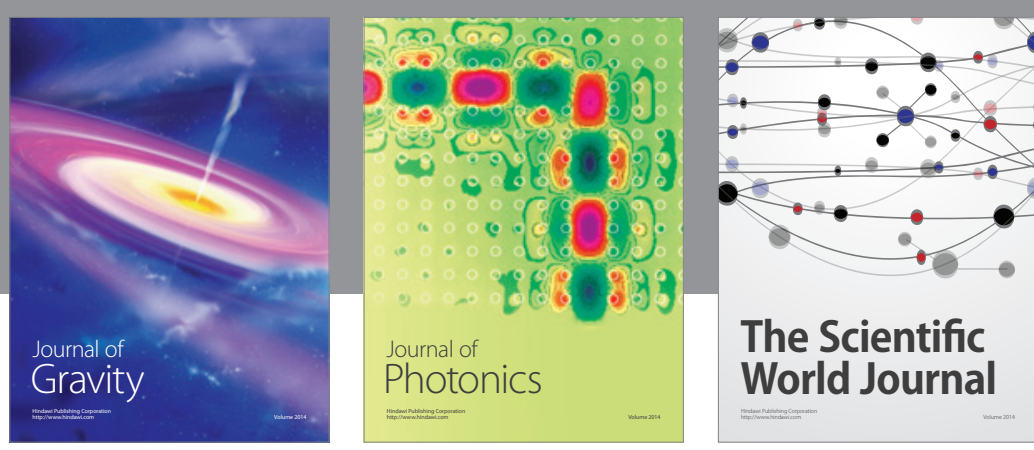

The Scientific World Journal
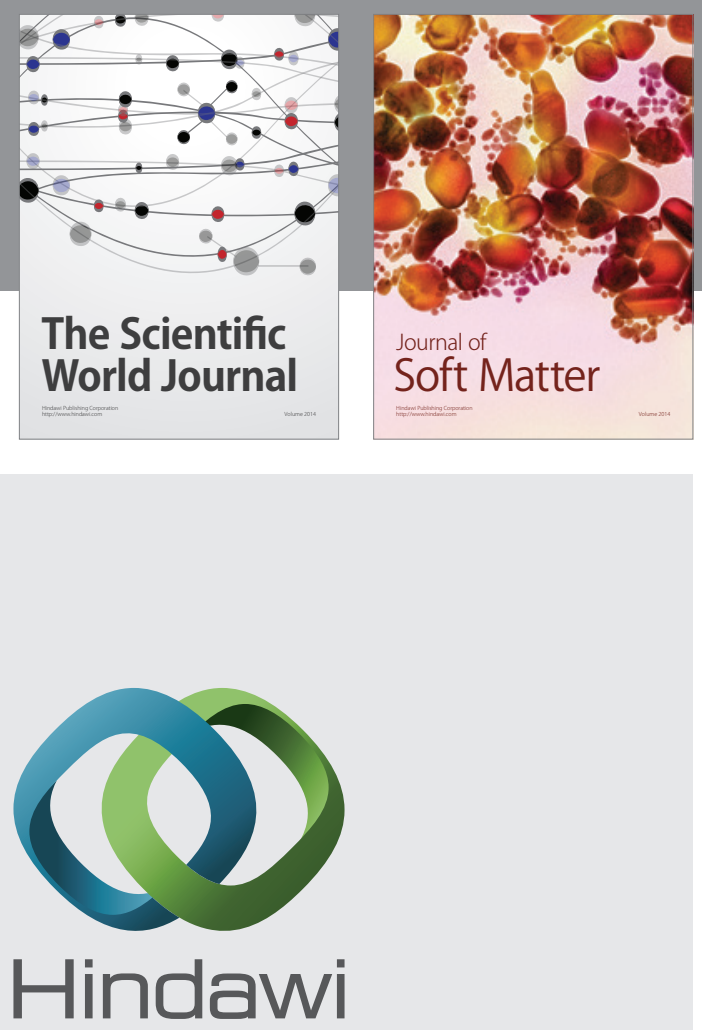

Submit your manuscripts at

http://www.hindawi.com

nternational Journal of

Statistical Mechanics
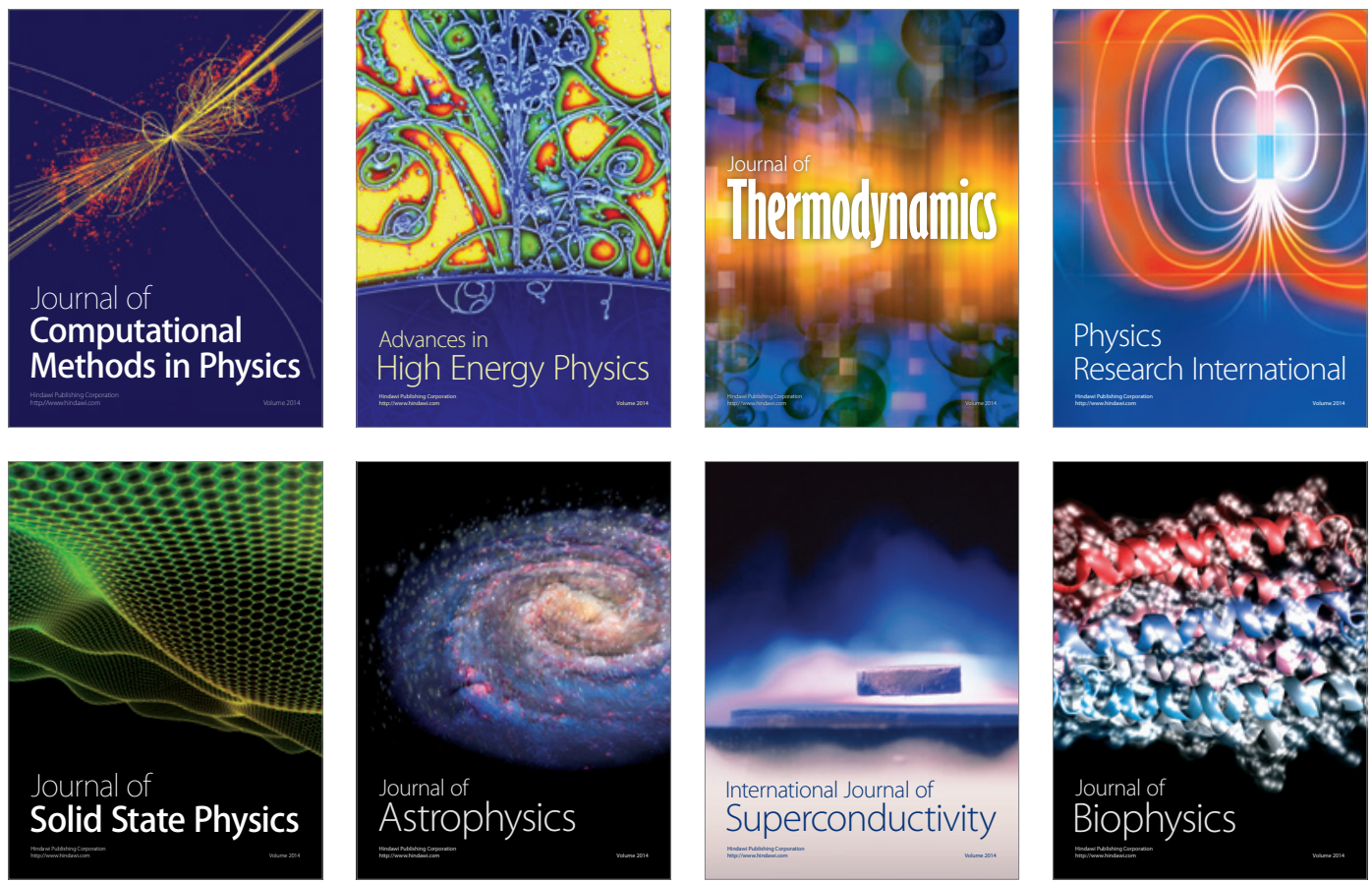
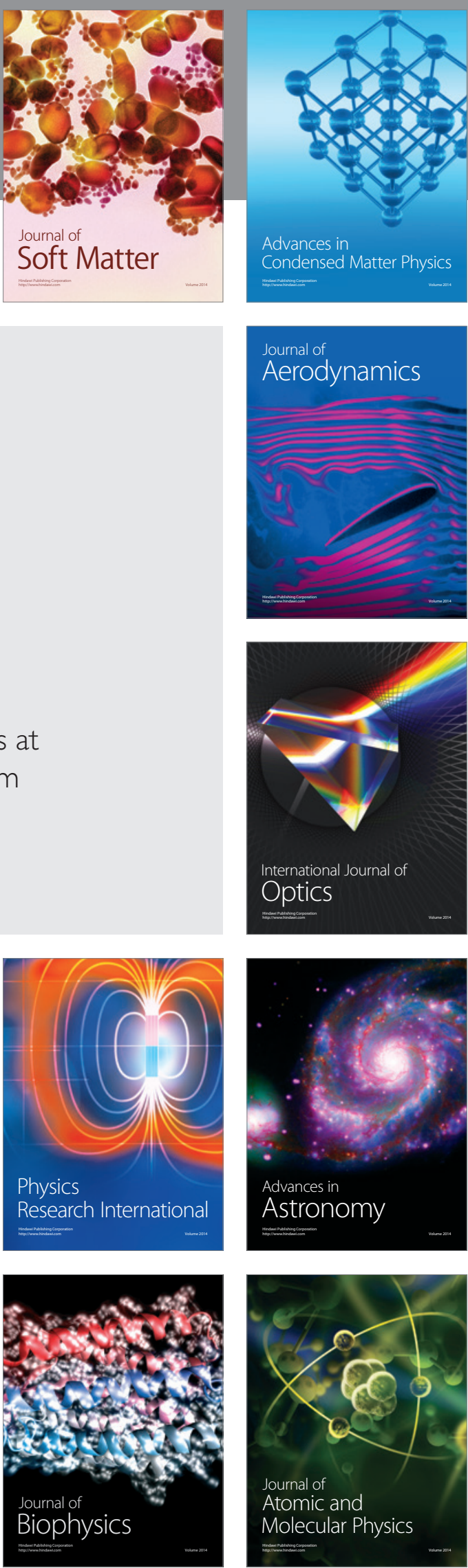